\title{
Global dynamics of an age-structured in-host viral infection model with humoral immunity
}

\section{Liangchen Li* and Rui Xu}

"Correspondence: IIc0610@126.com Institute of Applied Mathematics, Shijiazhuang Mechanical Engineering College, Heping Road, Shijiazhuang, China

\section{Springer}

\begin{abstract}
An age-structured in-host viral infection model with humoral immunity, consisting of partial differential and ordinary differential equations, is investigated. By calculation we get the basic reproduction number $\Re_{0}$ and the immune-activated reproduction number $\Re_{1}$. By analyzing the characteristic equations, the local stability of an infection-free steady state, an immune-inactivated infected steady state and an immune-activated infected steady state of the model is established. By using suitable Lyapunov functionals and LaSalle's invariance principle, it is proved that if $\Re_{0}<1$, the infection-free steady state is globally asymptotically stable; if $\Re_{1}<1<\Re_{0}$, the immune-inactivated infected steady state is globally asymptotically stable; and if $\Re_{1}>1$, the immune-activated infected steady state is globally asymptotically stable. Numerical simulations are carried out to illustrate the theoretical results.
\end{abstract}

MSC: 92D30; 34D23; 34K20

Keywords: age of infection; stability; Lyapunov functionals; LaSalle's invariance principle

\section{Introduction}

The adaptive immune system, also known as the acquired immune system, is mainly composed of two parts, cellular immunity and humoral immunity. Humoral immunity is mediated by macromolecules such as antibodies, complement proteins, and certain antimicrobial peptides. These macromolecules are produced by a special kind of leukocyte, the $\mathrm{B}$ lymphocyte. The principal function of B cells is to make these macromolecules against soluble antigens. So humoral immunity can be more effective than cellular immunity in some infections, such as malaria [1]. To investigate the role of humoral immunity in infection, many authors have presented and developed mathematical models [2-7]. Murase et al. introduced a basic in-host viral model with humoral immunity response in [5]:

$$
\begin{aligned}
& \frac{d x(t)}{d t}=s-d x(t)-\beta x(t) v(t), \\
& \frac{d y(t)}{d t}=\beta x(t) v(t)-\delta y(t),
\end{aligned}
$$

(c) $2016 \mathrm{Li}$ and $\mathrm{Xu}$. This article is distributed under the terms of the Creative Commons Attribution 4.0 International License (http://creativecommons.org/licenses/by/4.0/), which permits unrestricted use, distribution, and reproduction in any medium, provided you give appropriate credit to the original author(s) and the source, provide a link to the Creative Commons license, and indicate if changes were made. 


$$
\begin{aligned}
& \frac{d v(t)}{d t}=k y(t)-u v(t)-p v(t) z(t), \\
& \frac{d z(t)}{d t}=c v(t) z(t)-b z(t) .
\end{aligned}
$$

In system (1.1), $x(t), y(t), v(t)$, and $z(t)$ are the densities of uninfected cells, infected cells, viruses, and B cells at time $t$, respectively. $s$ and $d$ are the birth rate and death rate of uninfected cells. $\beta$ is the infection rate. $k$ is the average virion production rate of infected cells. $\delta$ is the death rate of infected cells, $u$ is the death rate of the virus. $c, b$, and $p$ are the birth rate, death rate, and neutralized rate of B cells, respectively.

In [8] Nelson et al. suggested that the death rate of infected cells should vary over their life span and the virion production rate is initially low and increases with the age of infection. Further, they introduced an age-structured HIV infection model taking the following form:

$$
\begin{aligned}
& \dot{x}(t)=s-d x(t)-\beta x(t) v(t), \\
& \frac{\partial y(a, t)}{\partial a}+\frac{\partial y(a, t)}{\partial t}=-\delta(a) y(a, t), \\
& \dot{v}(t)=\int_{0}^{\infty} k(a) y(a, t) d a-u v(t) .
\end{aligned}
$$

In system (1.2), $x(t)$ and $v(t)$ denote the densities of uninfected target $\mathrm{T}$ cells and infectious free virions at time $t$, respectively. $y(a, t)$ denotes the density of infected T cells of infection age $a$ at time $t$. The definitions of the various parameters in system (1.2) are listed in Table 1 .

In their model, the production rate of viral particles and the death rate of productively infected cells are allowed to vary and depend on two general functions of age. These assumptions are reasonable and the supporting evidence can be found in the recent research of Reilly et al. and Gilchrist et al. in [9, 10]. Nelson et al. analyzed the local stability of the equilibria of the age-structured HIV infection model when they introduced it. Later Huang et al. established the global asymptotic stability of the equilibria by using suitable Lyapunov functionals and Lasalle's invariance principle in [11]. Recently, in [12] Wang et $a l$. analyzed an age-structured HIV infection model with saturation infection rate, their result is an extension to the work of Nelson et al. and Huang et al.

Motivated by the basic in-host viral model with humoral immunity response introduced in [5] and work of Nelson et al. in [8], in this paper, we study an age-structured in-host

Table 1 Biological definitions of parameters

\begin{tabular}{ll}
\hline Parameter & Biological definition \\
\hline$a$ & Age of infection \\
$s$ & Recruitment rate of healthy T cells \\
$d$ & Per capita death rate of uninfected cells \\
$\beta$ & Rate at which an uninfected cell becomes \\
& infected by an infectious virus \\
$k(a)$ & Virion production rate of an infected cell with age $a$ \\
$\delta(a)$ & Age-dependent per capita death rate of infected cells \\
$u$ & Clearance rate of virions \\
\hline
\end{tabular}


viral infection model with humoral immunity, the model takes the following form:

$$
\begin{aligned}
& \dot{x}(t)=s-d x(t)-\beta x(t) v(t), \\
& \frac{\partial y(a, t)}{\partial t}+\frac{\partial y(a, t)}{\partial a}=-\delta(a) y(a, t), \\
& \dot{v}(t)=\int_{0}^{\infty} k(a) y(a, t) d a-u v(t)-p v(t) z(t), \\
& \dot{z}(t)=c v(t) z(t)-b z(t),
\end{aligned}
$$

with boundary condition

$$
y(0, t)=\beta x(t) v(t)
$$

and initial condition

$$
x(0)=x_{s}, \quad y(a, 0)=y_{s}(a), \quad v(0)=v_{s}, \quad z(0)=z_{s} .
$$

In system (1.3), $y(a, t)$ is the density of infected cells of infection age $a$ at time $t, k(a)$ is the virion production rate of infected cells with infection age $a, \delta(a)$ is the age-dependent per capita death rate of infected cells. The definitions of $x(t), v(t), z(t)$, and the other parameters are the same as in system (1.1). To make the model biologically meaningful, we assume:

(H1) $a \geq 0, s>0, d>0, \beta>0, u>0, p>0, c>0, b>0$.

(H2) $\delta(a)$ is bounded and $\delta(a)>\delta_{\min }$ for some positive constant $\delta_{\min }$ for all $a \geq 0$.

(H3) $k(a)$ is bounded and there exists a maximum age $a^{+}$for the virion production such that $k(a)>0$ for $0<a<a^{+}, k(a)=0$ for $a \geq a^{+}$.

(H4) $x_{s}>0, y_{s}(a) \geq 0, v_{s}>0, z_{s} \geq 0$.

According to (H1)-(H4), it is easy to see that system (1.3) with boundary condition (1.4) and initial condition (1.5) has a unique nonnegative solution.

\section{Local stability}

Denote

$$
N=\int_{0}^{\infty} k(a) \sigma(a) d a
$$

where

$$
\sigma(a)=e^{-\int_{0}^{a} \delta(\varepsilon) d \varepsilon}
$$

$N$ stands for the total number of viral particles produced by an infected cell in its lifespan. According to (H3), $N$ also takes the form

$$
N=\int_{0}^{a^{+}} k(a) \sigma(a) d a
$$

By calculation we know that the infection-free steady state of system (1.3) is $E_{0}(s / d, 0,0,0)$. If the basic reproduction number $\Re_{0}=\beta s N / d u>1$, there exists an immune-inactivated 
infected steady state $E_{1}^{*}\left(x_{1}^{*}, y_{1}^{*}(a), v_{1}^{*}, 0\right)$, in which

$$
x_{1}^{*}=\frac{u}{\beta N}, \quad y_{1}^{*}(a)=\frac{(\beta s N-d u) \sigma(a)}{\beta N}, \quad v_{1}^{*}=\frac{\beta s N-d u}{\beta u} .
$$

If the immune-activated reproduction number $\Re_{1}=\beta c s N /(c d u+\beta b u)>1$, there exists an immune-activated infected steady state $E_{2}^{*}\left(x_{2}^{*}, y_{2}^{*}(a), v_{2}^{*}, z_{2}^{*}\right)$, in which

$$
x_{2}^{*}=\frac{c s}{c d+\beta b}, \quad y_{2}^{*}(a)=\frac{\beta b s \sigma(a)}{c d+\beta b}, \quad v_{2}^{*}=\frac{b}{c}, \quad z_{2}^{*}=\frac{\beta c s N-c d u-\beta b u}{p(c d+\beta b)} .
$$

Theorem 2.1 The infection-free steady state $E_{0}$ is locally asymptotically stable if $\Re_{0}<1$.

Proof Linearizing system (1.3) about $E_{0}$ and defining the perturbation variables

$$
x_{1}(t)=x(t)-\frac{s}{d}, \quad y_{1}(a, t)=y(a, t), \quad v_{1}(t)=v(t), \quad z_{1}(t)=z(t),
$$

we obtain

$$
\begin{aligned}
& \dot{x}_{1}(t)=-d x_{1}(t)-\frac{\beta s}{d} v_{1}(t), \\
& \frac{\partial y_{1}(a, t)}{\partial a}+\frac{\partial y_{1}(a, t)}{\partial t}=-\delta(a) y_{1}(a, t), \\
& \dot{v}_{1}(t)=\int_{0}^{\infty} k(a) y_{1}(a, t) d a-u v_{1}(t), \\
& \dot{z}_{1}(t)=-b z_{1}(t),
\end{aligned}
$$

and

$$
y_{1}(0, t)=\frac{\beta s}{d} v_{1}(t) .
$$

Look for non-trivial solutions of (2.2) and (2.3) of the form

$$
x_{1}(t)=c_{11} e^{\lambda t}, \quad y_{1}(a, t)=y_{1}^{0}(a) e^{\lambda t}, \quad v_{1}(t)=c_{21} e^{\lambda t}, \quad z_{1}(t)=c_{31} e^{\lambda t} .
$$

Substituting (2.4) into (2.2) and (2.3), it follows that

$$
\begin{aligned}
& (\lambda+d) c_{11}=-\frac{\beta s}{d} c_{21}, \\
& \frac{d y_{1}^{0}(a)}{d a}=-(\delta(a)+\lambda) y_{1}^{0}(a), \\
& (\lambda+u) c_{21}=\int_{0}^{\infty} k(a) y_{1}^{0}(a) d a, \\
& c_{31} \lambda=-b c_{31}, \\
& y_{1}^{0}(0)=\frac{\beta s}{d} c_{21} .
\end{aligned}
$$

Integrating the second equation of (2.5) from 0 to $a$ yields

$$
y_{1}^{0}(a)=y_{1}^{0}(0) e^{-\int_{0}^{a}(\lambda+\delta(\varepsilon)) d \varepsilon} .
$$


We derive from the fifth equation of (2.5) and (2.6) that

$$
y_{1}^{0}(a)=\frac{\beta s}{d} c_{21} e^{-\int_{0}^{a}(\lambda+\delta(\varepsilon)) d \varepsilon} .
$$

Then substituting (2.7) and the fourth equation of (2.5) into the third equation of (2.5), we obtain the characteristic equation

$$
(\lambda+b)\left(\frac{N}{\int_{0}^{\infty} k(a) e^{-\int_{0}^{a}(\lambda+\delta(\varepsilon)) d \varepsilon} d a} \cdot \frac{\lambda+u}{u}-\Re_{0}\right)=0 .
$$

Clearly, $\lambda=-b$ is a negative real root of equation (2.8). We claim that if $\Re_{0}<1$, all roots of equation (2.8) have negative real parts. Otherwise, equation (2.8) has at least one root satisfying $\operatorname{Re} \lambda \geq 0$, in this case

$$
\begin{aligned}
\Re_{0} & =\left|\frac{N}{\int_{0}^{\infty} k(a) e^{-\int_{0}^{a}(\lambda+\delta(\varepsilon)) d \varepsilon} d a} \cdot \frac{\lambda+u}{u}\right| \\
& =\left|\frac{\int_{0}^{\infty} k(a) e^{-\int_{0}^{a} \delta(\varepsilon) d \varepsilon} d a}{\int_{0}^{\infty} e^{-a \lambda} k(a) e^{-\int_{0}^{a} \delta(\varepsilon) d \varepsilon} d a}\right| \cdot\left|\frac{\lambda+u}{u}\right| \geq 1 .
\end{aligned}
$$

It contradicts with $\Re_{0}<1$. Therefore, all roots of equation (2.8) have negative real parts. Accordingly, $E_{0}$ is locally asymptotically stable if $\Re_{0}<1$.

Theorem 2.2 The immune-inactivated infected steady state $E_{1}^{*}$ is locally asymptotically stable if $\Re_{1}<1<\Re_{0}$.

Proof Linearizing system (1.3) about $E_{1}^{*}$ and defining the perturbation variables

$$
x_{2}(t)=x(t)-x_{1}^{*}, \quad y_{2}(a, t)=y(a, t)-y_{1}^{*}(a), \quad v_{2}(t)=v(t)-v_{1}^{*}, \quad z_{2}(t)=z(t),
$$

we obtain

$$
\begin{aligned}
& \dot{x}_{2}(t)=\left(-d-\beta v_{1}^{*}\right) x_{2}(t)-\beta x_{1}^{*} v_{2}(t), \\
& \frac{\partial y_{2}(a, t)}{\partial a}+\frac{\partial y_{2}(a, t)}{\partial t}=-\delta(a) y_{2}(a, t), \\
& \dot{v}_{2}(t)=\int_{0}^{\infty} k(a) y_{2}(a, t) d a-u v_{2}(t)-p v_{1}^{*} z_{2}(t), \\
& \dot{z}_{2}(t)=\left(c v_{1}^{*}-b\right) z_{2}(t),
\end{aligned}
$$

and

$$
y_{2}(0, t)=\beta x_{1}^{*} v_{2}(t)+\beta v_{1}^{*} x_{2}(t) .
$$

We look for non-trivial solutions of (2.9) and (2.10) of the form

$$
x_{2}(t)=c_{12} e^{\lambda t}, \quad y_{2}(a, t)=y_{2}^{0}(a) e^{\lambda t}, \quad v_{2}(t)=c_{22} e^{\lambda t}, \quad z_{2}(t)=c_{32} e^{\lambda t} .
$$


By using a similar method to the proof of Theorem 2.1, we obtain the characteristic equation

$$
\left(\frac{\lambda+u}{u}-\frac{\lambda+d}{\lambda+d+\beta v_{1}^{*}} \cdot \frac{\int_{0}^{\infty} k(a) e^{-\int_{0}^{a}(\delta(\varepsilon)+\lambda) d \varepsilon} d a}{\int_{0}^{\infty} k(a) e^{-\int_{0}^{a} \delta(\varepsilon) d \varepsilon} d a}\right)\left(\lambda-c v_{1}^{*}+b\right)=0
$$

Obviously, $\lambda=c v_{1}^{*}-b=\left(\Re_{1}-1\right)(c d+\beta b) / \beta$ is a root of equation (2.12), and it is negative when $\Re_{1}<1$. We claim that if $\Re_{1}<1<\Re_{0}$, all roots of equation (2.12) have negative real parts. Otherwise, equation (2.12) has at least one root satisfying $\operatorname{Re} \lambda \geq 0$, in this case

$$
\frac{\lambda+u}{u}=\frac{\lambda+d}{\lambda+d+\beta v_{1}^{*}} \cdot \frac{\int_{0}^{\infty} k(a) e^{-\int_{0}^{a}(\delta(\varepsilon)+\lambda) d \varepsilon} d a}{\int_{0}^{\infty} k(a) e^{-\int_{0}^{a} \delta(\varepsilon) d \varepsilon} d a} .
$$

However,

$$
\begin{aligned}
& \left|\frac{\lambda+u}{u}\right| \geq 1, \\
& \left|\frac{\lambda+d}{\lambda+d+\beta v_{1}^{*}} \cdot \frac{\int_{0}^{\infty} k(a) e^{-\int_{0}^{a}(\delta(\varepsilon)+\lambda) d \varepsilon} d a}{\int_{0}^{\infty} k(a) e^{-\int_{0}^{a} \delta(\varepsilon) d \varepsilon} d a}\right| \\
& \quad=\left|\frac{\lambda+d}{\lambda+d+\beta v_{1}^{*}}\right| \cdot\left|\frac{\int_{0}^{\infty} k(a) e^{-\int_{0}^{a}(\delta(\varepsilon)+\lambda) d \varepsilon} d a}{\int_{0}^{\infty} k(a) e^{-\int_{0}^{a} \delta(\varepsilon) d \varepsilon} d a}\right|<1 .
\end{aligned}
$$

A contradiction occurs. Thus, all roots of equation (2.12) have negative real parts, $E_{1}^{*}$ is locally asymptotically stable if $\Re_{1}<1<\Re_{0}$.

Theorem 2.3 The immune-activated infected steady state $E_{2}^{*}$ is locally asymptotically stable if $\Re_{1}>1$.

Proof Linearizing system (1.3) about $E_{1}^{*}$ and defining the perturbation variables

$$
\begin{array}{ll}
x_{3}(t)=x(t)-x_{2}^{*}, & y_{3}(a, t)=y(a, t)-y_{2}^{*}(a), \\
v_{3}(t)=v(t)-v_{2}^{*}, & z_{3}(t)=z(t)-z_{2}^{*},
\end{array}
$$

we obtain

$$
\begin{aligned}
& \dot{x}_{3}(t)=\left(-d-\beta v_{2}^{*}\right) x_{3}(t)-\beta x_{2}^{*} v_{3}(t), \\
& \frac{\partial y_{3}(a, t)}{\partial a}+\frac{\partial y_{3}(a, t)}{\partial t}=-\delta(a) y_{3}(a, t), \\
& \dot{v}_{3}(t)=\int_{0}^{\infty} k(a) y_{3}(a, t) d a-\left(u+p z_{2}^{*}\right) v_{3}(t)-p v_{2}^{*} z_{3}(t), \\
& \dot{z}_{3}(t)=c z_{2}^{*} v_{3}(t),
\end{aligned}
$$

and

$$
y_{3}(0, t)=\beta x_{2}^{*} \nu_{3}(t)+\beta v_{2}^{*} x_{3}(t) .
$$


We look for non-trivial solutions of (2.13) and (2.14) of the form

$$
x_{3}(t)=c_{13} e^{\lambda t}, \quad y_{3}(a, t)=y_{3}^{0}(a) e^{\lambda t}, \quad v_{3}(t)=c_{23} e^{\lambda t}, \quad z_{3}(t)=c_{33} e^{\lambda t} .
$$

Substituting (2.15) into (2.13) and (2.14), it follows that

$$
\begin{aligned}
& c_{13} \lambda=\left(-d-\beta v_{2}^{*}\right) c_{13}-\beta x_{2}^{*} c_{23}, \\
& \frac{d y_{3}^{0}(a)}{d a}=-(\delta(a)+\lambda) y_{3}^{0}(a), \\
& c_{23} \lambda=\int_{0}^{\infty} k(a) y_{3}^{0}(a) d a-\left(u+p z_{2}^{*}\right) c_{23}-p v_{2}^{*} c_{33}, \\
& c_{33} \lambda=c z_{2}^{*} c_{23}, \\
& y_{3}^{0}(0)=\beta x_{2}^{*} c_{23}+\beta v_{2}^{*} c_{13} .
\end{aligned}
$$

We derive from the second and the fifth equations of (2.16) that

$$
y_{3}^{0}(a)=\left(\beta x_{2}^{*} c_{23}+\beta v_{2}^{*} c_{13}\right) e^{-\int_{0}^{a}(\delta(\varepsilon)+\lambda) d \varepsilon} .
$$

Substituting the first and the fourth equations of (2.16) into the third equation of (2.16), we get the characteristic equation

$$
\begin{gathered}
\lambda\left(\lambda+u+p z_{2}^{*}\right)\left(\lambda+d+\beta v_{2}^{*}\right)+c p v_{2}^{*} z_{2}^{*}\left(\lambda+d+\beta v_{2}^{*}\right) \\
\quad=\lambda(\lambda+d) \beta x_{2}^{*} \int_{0}^{\infty} k(a) e^{-\int_{0}^{a}(\delta(\varepsilon)+\lambda) d \varepsilon} d a .
\end{gathered}
$$

Noting that $\lambda=0$ and $\lambda=-d-\beta v_{2}^{*}$ are not roots of equation (2.18), (2.18) can also be written as

$$
\lambda+u+p z_{2}^{*}+\frac{c p v_{2}^{*} z_{2}^{*}}{\lambda}=\frac{(\lambda+d) \beta x_{2}^{*}}{\lambda+d+\beta v_{2}^{*}} \int_{0}^{\infty} k(a) e^{-\int_{0}^{a}(\delta(\varepsilon)+\lambda) d \varepsilon} d a
$$

Substituting $x_{2}^{*}=c s /(c d+\beta b), v_{2}^{*}=b / c, z_{2}^{*}=(\beta c s N-c d u-\beta b u) / p(c d+\beta b)$ into (2.19), we have

$$
\frac{\beta c s N}{c d+\beta b}+\frac{\lambda^{2}+p b z_{2}^{*}}{\lambda}=\frac{\lambda+d}{\lambda+d+\beta v_{2}^{*}} \frac{\beta c s}{c d+\beta b} \int_{0}^{\infty} k(a) e^{-\int_{0}^{a}(\delta(\varepsilon)+\lambda) d \varepsilon} d a .
$$

We claim that if $\Re_{1}>1$, all roots of equation (2.20) have negative real parts. Otherwise, equation (2.20) has at least one root satisfying $\lambda=\alpha+\gamma i(\alpha>0$ or $\alpha=0, \gamma \neq 0)$, in this case

$$
\begin{aligned}
& \left|\frac{\lambda+d}{\lambda+d+\beta v_{2}^{*}} \frac{\beta c s}{c d+\beta b} \int_{0}^{\infty} k(a) e^{-\int_{0}^{a}(\delta(\varepsilon)+\lambda) d \varepsilon} d a\right| \\
& \quad=\frac{\beta c s}{c d+\beta b}\left|\frac{\lambda+d}{\lambda+d+\beta v_{2}^{*}}\right|\left|\int_{0}^{\infty} k(a) e^{-\int_{0}^{a}(\delta(\varepsilon)+\lambda) d \varepsilon} d a\right|<\frac{\beta c s N}{c d+\beta b}
\end{aligned}
$$


while

$$
\begin{aligned}
\left|\frac{\beta c s N}{c d+\beta b}+\frac{\lambda^{2}+p b z^{*}}{\lambda}\right| & =\left|\frac{\beta c s N}{c d+\beta b}+\frac{\alpha^{2}-\gamma^{2}+2 \alpha \gamma i+p b z^{*}}{\alpha+\gamma i}\right| \\
& =\left|\frac{\beta c s N}{c d+\beta b}+\frac{\alpha\left(\alpha^{2}+\gamma^{2}+p b z^{*}\right)+\left(\alpha^{2} \gamma+\gamma^{3}-p b z^{*} \gamma\right) i}{\alpha^{2}+\gamma^{2}}\right| \\
& \geq \frac{\beta c s N}{c d+\beta b} .
\end{aligned}
$$

The contradiction is obvious. Thus, all roots of equation (2.20) have negative real parts, $E_{2}^{*}$ is locally asymptotically stable if $\Re_{1}>1$.

\section{Global stability}

In this section, we study the global asymptotic stability of each steady state of system (1.3). The strategy of proofs is to use Lyapunov functionals.

Theorem 3.1 The infection-free steady state $E_{0}$ is globally asymptotically stable if $\Re_{0}<1$.

Proof Denote

$$
f(a):=\int_{a}^{\infty} k(\theta) e^{-\int_{a}^{\theta} \delta(\varepsilon) d \varepsilon} d \theta \quad\left(=\int_{a}^{a^{+}} k(\theta) e^{-\int_{a}^{\theta} \delta(\varepsilon) d \varepsilon} d \theta\right)
$$

Note that $f(a)>0$ for all $0<a<a^{+}$. It is easy to show from (3.1) that $f(0)=N, f\left(a^{+}\right)=0$. Further, the derivative of $f(a)$ satisfies

$$
f^{\prime}(a)=\delta(a) f(a)-k(a)
$$

Let $(x(t), y(a, t), v(t), z(t))$ be any solution of system (1.3) with boundary condition (1.4) and initial condition (1.5). Define

$$
V_{1}(t)=\left(x(t)-x_{0}-x_{0} \ln \frac{x(t)}{x_{0}}\right)+\frac{1}{N} \int_{0}^{a^{+}} f(a) y(a, t) d a+\frac{1}{N} v(t)+\frac{p}{c N} z(t) .
$$

It is easy to see that $V_{1}(t)$ is nonnegative and $E_{0}$ is a global minimum of $V_{1}(t)$. Calculating the derivative of $V_{1}(t)$ along the solutions of system (1.3), we have

$$
\frac{d V_{1}(t)}{d t}=\left(1-\frac{x_{0}}{x}\right) \frac{d x(t)}{d t}+\frac{1}{N} \int_{0}^{a^{+}} f(a) \frac{\partial y(a, t)}{\partial t} d a+\frac{1}{N} \frac{d v(t)}{d t}+\frac{p}{c N} \frac{d z(t)}{d t}
$$

Substituting $s=d x_{0}$ and (3.2) into (3.3), we get

$$
\begin{aligned}
\frac{d V_{1}(t)}{d t}= & \left(1-\frac{x_{0}}{x(t)}\right)\left(d x_{0}-d x(t)-\beta x(t) v(t)\right) \\
& -\frac{1}{N} \int_{0}^{a^{+}} f(a)\left(\frac{\partial y(a, t)}{\partial a}+\delta(a) y(a, t)\right) d a \\
& +\frac{1}{N}\left(\int_{0}^{a^{+}} k(a) y(a, t) d a-u v(t)-p v(t) z(t)\right)
\end{aligned}
$$




$$
\begin{aligned}
& +\frac{p}{c N}(c v(t) z(t)-b z(t)) \\
= & -\frac{d}{x(t)}\left(x(t)-x_{0}\right)^{2}+\beta x_{0} v(t)-\beta x(t) v(t) \\
& -\frac{1}{N} \int_{0}^{a^{+}} f(a)\left(\frac{\partial y(a, t)}{\partial a}+\delta(a) y(a, t)\right) d a \\
& +\frac{1}{N} \int_{0}^{a^{+}} k(a) y(a, t) d a-\frac{u}{N} v(t)-\frac{b p}{c N} z(t) .
\end{aligned}
$$

Using integration by parts and $f(0)=N, f\left(a^{+}\right)=0, y(0, t)=\beta x(t) v(t)$, we have

$$
\begin{aligned}
\int_{0}^{a^{+}} f(a) \frac{\partial y(a, t)}{\partial a} d a & =f\left(a^{+}\right) y\left(a^{+}, t\right)-f(0) y(0, t)-\int_{0}^{a^{+}} f^{\prime}(a) y(a, t) d a \\
& =-\beta N x(t) v(t)-\int_{0}^{a^{+}} f^{\prime}(a) y(a, t) d a .
\end{aligned}
$$

Substituting (3.5) into (3.4) yields

$$
\begin{aligned}
\frac{d V_{1}(t)}{d t}= & -\frac{d}{x(t)}\left(x(t)-x_{0}\right)^{2}+\beta x_{0} v(t)-\frac{1}{N} \int_{0}^{a^{+}} f(a) \delta(a) y(a, t) d a \\
& +\frac{1}{N} \int_{0}^{a^{+}}(\delta(a) f(a)-k(a)) y(a, t) d a+\frac{1}{N} \int_{0}^{a^{+}} k(a) y(a, t) d a \\
& -\frac{u}{N} v(t)-\frac{b p}{c N} z(t) \\
= & -\frac{d}{x(t)}\left(x(t)-x_{0}\right)^{2}+\frac{u}{N}\left(\Re_{0}-1\right) v(t)-\frac{b p}{c N} z(t) .
\end{aligned}
$$

Therefore, $\Re_{0}<1$ ensures that $V_{1}^{\prime}(t) \leq 0$ holds true. By Theorem 5.3.1 in [13], solutions limit to $\mathcal{M}$, the largest invariant subset of $\left\{V_{1}^{\prime}(t)=0\right\}$. Clearly, $V_{1}^{\prime}(t)=0$ if and only if $x(t)=$ $x_{0}, v(t)=0, z(t)=0$. Noting that $\mathcal{M}$ is invariant, for each element in $\mathcal{M}$, we have $v(t)=0$, $z(t)=0, v^{\prime}(t)=0$. We therefore derive from the third equation of system (1.3) that $y(a, t)=$ 0 . Hence, $V_{1}^{\prime}(t)=0$ if and only if $(x(t), y(a, t), v(t), z(t))=\left(x_{0}, 0,0,0\right)$. Accordingly the global asymptotic stability of $E_{0}$ follows from LaSalle's invariance principle. This completes the proof.

Theorem 3.2 The immune-inactivated infected steady state $E_{1}^{*}$ is globally asymptotically stable if $\Re_{1}<1<\Re_{0}$.

Proof Let $(x(t), y(a, t), v(t), z(t))$ be any solution of system (1.3) with boundary condition (1.4) and initial condition (1.5). Define

$$
\begin{aligned}
V_{2}(t)= & \left(x(t)-x_{1}^{*}-x_{1}^{*} \ln \frac{x(t)}{x_{1}^{*}}\right) \\
& +\frac{1}{N} \int_{0}^{a^{+}} f(a) y_{1}^{*}(a)\left(\frac{y(a, t)}{y_{1}^{*}(a)}-1-\ln \frac{y(a, t)}{y_{1}^{*}(a)}\right) d a \\
& +\frac{1}{N}\left(v(t)-v_{1}^{*}-v_{1}^{*} \ln \frac{v(t)}{v_{1}}\right)+\frac{p}{c N} z(t),
\end{aligned}
$$


where $f(a)$ is defined in (3.1). It is easy to see that $V_{2}(t)$ is nonnegative and $E_{1}^{*}$ is a global minimum. Calculating the derivative of $V_{2}(t)$ along the solutions of system (1.3), we have

$$
\begin{aligned}
\frac{d V_{2}(t)}{d t}= & \left(1-\frac{x_{1}^{*}}{x(t)}\right)(s-d x(t)-\beta x(t) v(t)) \\
& +\frac{1}{N} \int_{0}^{a^{+}} f(a)\left(1-\frac{y_{1}^{*}(a)}{y(a, t)}\right) \frac{\partial y(a, t)}{\partial t} d a \\
& +\frac{1}{N}\left(1-\frac{v_{1}^{*}}{v(t)}\right)\left(\int_{0}^{\infty} k(a) y(a, t) d a-u v(t)-p v(t) z(t)\right) \\
& +\frac{p}{c N}(c v(t) z(t)-b z(t)) .
\end{aligned}
$$

By using $s=d x^{*}+\beta x^{*} v^{*}$, we have

$$
\begin{aligned}
\frac{d V_{2}(t)}{d t}= & \left(1-\frac{x_{1}^{*}}{x(t)}\right)\left(d x_{1}^{*}-d x(t)+\beta x_{1}^{*} v_{1}^{*}-\beta x(t) v(t)\right) \\
& -\frac{1}{N} \int_{0}^{a^{+}} f(a)\left(1-\frac{y_{1}^{*}(a)}{y(a, t)}\right)\left(\frac{\partial y(a, t)}{\partial a}+\delta(a) y(a, t)\right) d a \\
& +\frac{1}{N}\left(1-\frac{v_{1}^{*}}{v(t)}\right)\left(\int_{0}^{a^{+}} k(a) y(a, t) d a-u v(t)-p v(t) z(t)\right) \\
& +\frac{p}{c N}(c v(t) z(t)-b z(t)) \\
= & -\frac{d}{x(t)}\left(x_{1}^{*}-x(t)\right)^{2}+\left(1-\frac{x_{1}^{*}}{x(t)}\right)\left(\beta x_{1}^{*} v_{1}^{*}-\beta x(t) v(t)\right) \\
& -\frac{1}{N} \int_{0}^{a^{+}} f(a)\left(1-\frac{y_{1}^{*}(a)}{y(a, t)}\right)\left(\frac{\partial y(a, t)}{\partial a}+\delta(a) y(a, t)\right) d a \\
& +\frac{1}{N}\left(\int_{0}^{a^{+}} k(a) y(a, t) d a-u v(t)\right) \\
& -\frac{1}{N} \frac{v_{1}^{*}}{v(t)}\left(\int_{0}^{a^{+}} k(a) y(a, t) d a-u v(t)-p v(t) z(t)\right) \\
& -\frac{b p}{c N} z(t) .
\end{aligned}
$$

Note that

$$
\frac{d}{d a}\left(\frac{y(a, t)}{y_{1}^{*}(a)}-1-\ln \frac{y(a, t)}{y_{1}^{*}(a)}\right)=\left(1-\frac{y_{1}^{*}(a)}{y(a, t)}\right)\left(\frac{y_{a}(a, t)}{y_{1}^{*}(a)}-\frac{y(a, t) y_{1 a}^{*}(a)}{\left[y_{1}^{*}(a)\right]^{2}}\right),
$$

here $y_{a}(a, t)=\partial y(a, t) / \partial a$ and $y_{1 a}^{*}(a)=d y_{1}^{*}(a) / d a$. Since

$$
y_{a}^{*}(a)=-\delta(a) y^{*}(a)
$$

substituting (3.8) into (3.7) yields

$$
\begin{aligned}
\left(1-\frac{y_{1}^{*}(a)}{y(a, t)}\right) \frac{\partial y(a, t)}{\partial a}= & y_{1}^{*}(a) \frac{d}{d a}\left(\frac{y(a, t)}{y_{1}^{*}(a)}-1-\ln \frac{y(a, t)}{y_{1}^{*}(a)}\right) \\
& +\delta(a) y_{1}^{*}(a)-\delta(a) y(a, t) .
\end{aligned}
$$


Using integration by parts, it follows from (3.9) that

$$
\begin{aligned}
& \int_{0}^{a^{+}} f(a)\left(1-\frac{y_{1}^{*}(a)}{y(a, t)}\right) \frac{\partial y(a, t)}{\partial a} d a \\
& =f\left(a^{+}\right) y_{1}^{*}\left(a^{+}\right)\left(\frac{y\left(a^{+}, t\right)}{y_{1}^{*}\left(a^{+}\right)}-1-\ln \frac{y\left(a^{+}, t\right)}{y_{1}^{*}\left(a^{+}\right)}\right)-f(0) y_{1}^{*}(0)\left(\frac{y(0, t)}{y_{1}^{*}(0)}-1-\ln \frac{y(0, t)}{y_{1}^{*}(0)}\right) \\
& \quad-\int_{0}^{a^{+}}\left(\frac{y(a, t)}{y_{1}^{*}(a)}-1-\ln \frac{y(a, t)}{y_{1}^{*}(a)}\right)\left(f^{\prime}(a) y_{1}^{*}(a)+f(a) y_{1 a}^{*}(a)\right) d a \\
& \quad+\int_{0}^{a^{+}} f(a)\left(\delta(a) y_{1}^{*}(a)-\delta(a) y(a, t)\right) d a .
\end{aligned}
$$

Noting that

$$
\begin{aligned}
& f(0)=N, \quad f\left(a^{+}\right)=0, \\
& y_{1}^{*}(0)=\beta x_{1}^{*} v_{1}^{*}, \\
& y(0, t)=\beta x v, \\
& y_{1 a}^{*}(a)=-\delta(a) y_{1}^{*}(a), \\
& f^{\prime}(a)=\delta(a) f(a)-k(a),
\end{aligned}
$$

we have

$$
\begin{aligned}
& f(0) y_{1}^{*}(0)\left(\frac{y(0, t)}{y_{1}^{*}(0)}-1-\ln \frac{y(0, t)}{y_{1}^{*}(0)}\right)=N \beta x_{1}^{*} v_{1}^{*}\left(\frac{x v}{x_{1}^{*} v_{1}^{*}}-1-\ln \frac{x v}{x_{1}^{*} v_{1}^{*}}\right), \\
& f\left(a^{+}\right) y^{*}\left(a^{+}\right)\left(\frac{y\left(a^{+}, t\right)}{y_{1}^{*}\left(a^{+}\right)}-1-\ln \frac{y\left(a^{+}, t\right)}{y_{1}^{*}\left(a^{+}\right)}\right)=0, \\
& f^{\prime}(a) y_{1}^{*}(a)+f(a) y_{1 a}^{*}(a)=-k(a) y_{1}^{*}(a) .
\end{aligned}
$$

Further, we obtain

$$
\begin{gathered}
\int_{0}^{a^{+}} f(a)\left(1-\frac{y_{1}^{*}(a)}{y(a, t)}\right)\left(\frac{\partial y(a, t)}{\partial a}+\delta(a) y(a, t)\right) d a \\
=-N \beta x_{1}^{*} v_{1}^{*}\left(\frac{x v}{x_{1}^{*} v_{1}^{*}}-1-\ln \frac{x v}{x_{1}^{*} v_{1}^{*}}\right) \\
\quad+\int_{0}^{a^{+}}\left(\frac{y(a, t)}{y_{1}^{*}(a)}-1-\ln \frac{y(a, t)}{y_{1}^{*}(a)}\right) k(a) y_{1}^{*}(a) d a .
\end{gathered}
$$

We derive from (3.6) and (3.12) that

$$
\begin{aligned}
\frac{d V_{2}(t)}{d t}= & -\frac{d}{x(t)}\left(x_{1}^{*}-x(t)\right)^{2}+\left(1-\frac{x_{1}^{*}}{x(t)}\right)\left(\beta x_{1}^{*} v_{1}^{*}-\beta x(t) v(t)\right) \\
& +\beta x_{1}^{*} v_{1}^{*}\left(\frac{x v}{x_{1}^{*} v_{1}^{*}}-1-\ln \frac{x v}{x_{1}^{*} v_{1}^{*}}\right) \\
& -\frac{1}{N} \int_{0}^{a^{+}}\left(\frac{y(a, t)}{y_{1}^{*}(a)}-1-\ln \frac{y(a, t)}{y_{1}^{*}(a)}\right) k(a) y_{1}^{*}(a) d a
\end{aligned}
$$




$$
\begin{aligned}
& +\frac{1}{N}\left(\int_{0}^{a^{+}} k(a) y(a, t) d a-u v(t)\right) \\
& -\frac{1}{N} \frac{v_{1}^{*}}{v(t)}\left(\int_{0}^{a^{+}} k(a) y(a, t) d a-u v(t)-p v(t) z(t)\right)-\frac{b p}{c N} z(t) .
\end{aligned}
$$

Noting that $u v_{1}^{*}=\int_{0}^{\infty} k(a) y_{1}^{*}(a) d a=\beta x_{1}^{*} v_{1}^{*} N$, we have

$$
\begin{aligned}
\frac{d V_{2}(t)}{d t}= & -\frac{d}{x(t)}\left(x_{1}^{*}-x(t)\right)^{2} \\
& -\frac{1}{N} \int_{0}^{a^{+}}\left(\frac{v_{1}^{*} y(a, t)}{y_{1}^{*}(a) v(t)}-1-\ln \frac{v_{1}^{*} y(a, t)}{y_{1}^{*}(a) v(t)}\right) k(a) y_{1}^{*}(a) d a \\
& -\beta x_{1}^{*} v_{1}^{*}\left(\frac{x_{1}^{*}}{x(t)}-1-\ln \frac{x_{1}^{*}}{x(t)}\right)+\frac{p z(t)}{N}\left(v_{1}^{*}-\frac{b}{c}\right) .
\end{aligned}
$$

Since

$$
\frac{p z(t)}{N}\left(v_{1}^{*}-\frac{b}{c}\right)=\left(\frac{(\beta s N-d u)}{\beta u}-\frac{b}{c}\right) \frac{p z(t)}{N}=\left(\Re_{1}-1\right)(c d u+\beta b u) \frac{p z(t)}{\beta c u N},
$$

$V_{2}^{\prime}(t) \leq 0$ if $\Re_{1}<1<\Re_{0}$. By Theorem 5.3.1 in [13], solutions limit to $\mathcal{M}$, the largest invariant subset of $\left\{V_{2}^{\prime}(t)=0\right\}$. It is readily seen that $V_{2}^{\prime}=0$ if and only if $x(t)=x_{1}^{*}, y(a, t)=y_{1}^{*}(a)$, $v(t)=v_{1}^{*}, z(t)=0$. We have proved in Theorem 2.2 that $E_{1}^{*}$ is locally asymptotically stable if $\Re_{1}<1<\Re_{0}$, then the global asymptotic stability of $E_{1}^{*}$ follows.

Theorem 3.3 The immune-activated infected steady state $E_{2}^{*}$ is globally asymptotically stable if $\Re_{1}>1$.

Proof Let $(x(t), y(a, t), v(t), z(t))$ be any solution of system (1.3) with boundary condition (1.4) and initial condition (1.5). Define

$$
\begin{aligned}
V_{3}(t)= & \left(x(t)-x_{2}^{*}-x_{2}^{*} \ln \frac{x(t)}{x_{2}^{*}}\right) \\
& +\frac{1}{N} \int_{0}^{a^{+}} f(a) y_{2}^{*}(a)\left(\frac{y(a, t)}{y_{2}^{*}(a)}-1-\ln \frac{y(a, t)}{y_{2}^{*}(a)}\right) d a \\
& +\frac{1}{N}\left(v(t)-v_{2}^{*}-v_{2}^{*} \ln \frac{v(t)}{v_{2}^{*}}\right)+\frac{p}{c N}\left(z(t)-z_{2}^{*}-z_{2}^{*} \ln \frac{z(t)}{z_{2}^{*}}\right),
\end{aligned}
$$

where $f(a)$ is defined in (3.1). It is easy to see that $V_{3}(t)$ is nonnegative and $E_{2}^{*}$ is a global minimum. Calculating the derivative of $V_{3}(t)$ along the solutions of system (1.3), we have

$$
\begin{aligned}
\frac{d V_{3}(t)}{d t}= & \left(1-\frac{x_{2}^{*}}{x(t)}\right)(s-d x(t)-\beta x(t) v(t)) \\
& +\frac{1}{N} \int_{0}^{a^{+}} f(a)\left(1-\frac{y_{2}^{*}(a)}{y(a, t)}\right) \frac{\partial y(a, t)}{\partial t} d a \\
& +\frac{1}{N}\left(1-\frac{v_{2}^{*}}{v(t)}\right)\left(\int_{0}^{\infty} k(a) y(a, t) d a-u v(t)-p v(t) z(t)\right) \\
& +\frac{p}{c N}\left(1-\frac{z_{2}^{*}}{z(t)}\right)(c v(t) z(t)-b z(t)) .
\end{aligned}
$$


By using $s=d x_{2}^{*}+\beta x_{2}^{*} v_{2}^{*}$, we obtain

$$
\begin{aligned}
\frac{d V_{3}(t)}{d t}= & \left(1-\frac{x_{2}^{*}}{x(t)}\right)\left(d x_{2}^{*}+\beta x_{2}^{*} v_{2}^{*}-d x(t)-\beta x(t) v(t)\right) \\
& -\frac{1}{N} \int_{0}^{a^{+}} f(a)\left(1-\frac{y_{2}^{*}(a)}{y(a, t)}\right)\left(\frac{\partial y(a, t)}{\partial a}+\delta(a) y(a, t)\right) d a \\
& +\frac{1}{N}\left(1-\frac{v_{2}^{*}}{v(t)}\right)\left(\int_{0}^{\infty} k(a) y(a, t) d a-u v(t)-p v(t) z(t)\right) \\
& +\frac{p}{c N}\left(1-\frac{z_{2}^{*}}{z(t)}\right)(c v(t) z(t)-b z(t)) \\
= & -\frac{d}{x(t)}\left(x_{2}^{*}-x(t)\right)^{2}+\left(1-\frac{x_{2}^{*}}{x(t)}\right)\left(\beta x_{2}^{*} v_{2}^{*}-\beta x(t) v(t)\right) \\
& -\frac{1}{N} \int_{0}^{a^{+}} f(a)\left(1-\frac{y_{2}^{*}(a)}{y(a, t)}\right)\left(\frac{\partial y(a, t)}{\partial a}+\delta(a) y(a, t)\right) d a \\
& +\frac{1}{N}\left(\int_{0}^{\infty} k(a) y(a, t) d a-u v(t)\right) \\
& -\frac{1}{N} \frac{v_{2}^{*}}{v(t)}\left(\int_{0}^{\infty} k(a) y(a, t) d a-u v(t)-p v(t) z(t)\right) \\
& -\frac{p}{c N}\left(c v(t) z_{2}^{*}-b z_{2}^{*}\right)-\frac{b p}{c N} z(t) .
\end{aligned}
$$

By using a similar method to (3.7)-(3.12), we derive from (3.14) that

$$
\begin{aligned}
\frac{d V_{3}(t)}{d t}= & -\frac{d}{x(t)}\left(x_{2}^{*}-x(t)\right)^{2}+\left(1-\frac{x_{2}^{*}}{x(t)}\right)\left(\beta x_{2}^{*} v_{2}^{*}-\beta x(t) v(t)\right) \\
& +\beta x_{2}^{*} v_{2}^{*}\left(\frac{x v}{x_{2}^{*} v_{2}^{*}}-1-\ln \frac{x v}{x_{2}^{*} v_{2}^{*}}\right) \\
& -\frac{1}{N} \int_{0}^{a^{+}}\left(\frac{y(a, t)}{y_{2}^{*}(a)}-1-\ln \frac{y(a, t)}{y_{2}^{*}(a)}\right) k(a) y_{2}^{*}(a) d a \\
& +\frac{1}{N}\left(\int_{0}^{a^{+}} k(a) y(a, t) d a-u v(t)\right) \\
& -\frac{1}{N} \frac{v_{2}^{*}}{v(t)}\left(\int_{0}^{a^{+}} k(a) y(a, t) d a-u v(t)-p v(t) z(t)\right) \\
& -\frac{p}{c N}\left(c v(t) z_{2}^{*}-b z_{2}^{*}\right)-\frac{b p}{c N} z(t) .
\end{aligned}
$$

Substituting $u v_{2}^{*}=\int_{0}^{a^{+}} k(a) y_{2}^{*}(a) d a-p v_{2}^{*} z_{2}^{*}=\beta x_{2}^{*} v_{2}^{*} N-p v_{2}^{*} z_{2}^{*}$ and $v_{2}^{*}=b / c$ into (3.15), we have

$$
\begin{aligned}
\frac{d V_{3}(t)}{d t}= & -\frac{d}{x(t)}\left(x_{2}^{*}-x(t)\right)^{2}-\beta x_{2}^{*} v_{2}^{*}\left(\frac{x_{2}^{*}}{x(t)}-1-\ln \frac{x_{2}^{*}}{x(t)}\right) \\
& -\int_{0}^{a^{+}}\left(\frac{v_{2}^{*} y(a, t)}{y_{2}^{*}(a) v(t)}-1-\ln \frac{v_{2}^{*} y(a, t)}{y_{2}^{*}(a) v(t)}\right) k(a) y_{2}^{*}(a) d a .
\end{aligned}
$$

Therefore, $\Re_{1}>1$ ensures that $V_{3}^{\prime}(t) \leq 0$ holds true. By Theorem 5.3.1 in [13], solutions of (1.3) are limited to $\mathcal{M}$, the largest invariant subset of $\left\{V_{3}^{\prime}(t)=0\right\}$. Using a similar argument 
to that in the proof of Theorem 3.1, we know that the equality $V_{3}^{\prime}(t)=0$ holds true if and only if $x(t)=x_{2}^{*}, y(a, t)=y_{2}^{*}(a), v(t)=v_{2}^{*}, z(t)=z_{2}^{*}$. By LaSalle's invariance principle, the global asymptotic stability of $E_{2}^{*}$ follows. This completes the proof.

\section{Numerical simulations}

In this section, we give some numerical examples to illustrate the theoretical results obtained in Sections 2 and 3. The mean methods are discretizing the equations and using eulerian difference method. The value of integral terms is obtained by Simpson's rule.

Usually, there is an incubation period after the cells are infected. In this period, the death rate of the cells is not changed and the infected cells do not produce virion. Later there is an outbreak period, in this period the infected cells begin to produce virion and the production rate increases, and the death rate of infected cells also rapidly increases. After the outbreak period, the death rate and the virion production rate of the infected cells tend to be stable. Considering these properties we assume that the death rate $\delta(a)$ and the virion production rate $k(a)$ of the infected cells take the forms

$$
\delta(a)= \begin{cases}0.03, & 0 \leq a \leq 10 \\ 0.03+0.005(a-10) e^{-0.009(a-25)^{2}}, & 10<a \leq 40 \\ 0.05, & 40<a \leq a^{+}\end{cases}
$$

and

$$
k(a)= \begin{cases}0, & 0 \leq a \leq 10 \\ 30(a-10) e^{-0.05(a-25)^{2}}, & 10<a \leq 40 \\ 0.012, & 40<a \leq a^{+}\end{cases}
$$

where the maximum age of the cells $a^{+}$is supposed to be 300 days. The initial value is supposed to be

$$
x_{s}=800, \quad v_{s}=100, \quad y_{s}(a)=50(a+3) e^{-0.2(a+3)}, \quad z_{s}=300 .
$$

We will take a day as the unit time and plot the solutions of system (1.3) from 0 to 600 days.

Example 1 In system (1.3), we choose $s=20, d=0.03, \beta=0.00024, u=30, p=0.5, c=$ $0.000005, b=0.07$. In this case $\Re_{0}=0.5626<1$. By Theorem 3.1, the infection-free steady state $E_{0}(s / d, 0,0,0)$ is globally asymptotically stable, where $s / d=666.67$. (See Figure 1 .)

Example 2 In system (1.3), we choose $s=30, d=0.03, \beta=0.00024, u=18, p=0.5$, $c=0.000005, b=0.07$. In this case $\Re_{0}=1.4064>1, \Re_{1}=0.1153<1$. By Theorem 3.2, the immune-inactivated infected steady state $E_{1}^{*}\left(x_{1}^{*}, y_{1}^{*}(a), v_{1}^{*}, 0\right)$ is globally asymptotically stable, where $x_{1}^{*}=711.04, y_{1}^{*}(a)=8.66 \sigma(a), v_{1}^{*}=507.98$. (See Figure 2.)

Example 3 In system (1.3), we choose $s=20, d=0.03, \beta=0.00024, u=8, p=0.1, c=$ $0.01, b=2$. In this case $\Re_{1}=1.8186>1$. By Theorem 3.3, the immune-activated infected steady state $E_{2}^{*}\left(x_{2}^{*}, y_{2}^{*}(a), v_{2}^{*}, z_{2}^{*}\right)$ is globally asymptotically stable, where $x_{2}^{*}=574.41, y_{2}^{*}(a)=$ $2.76 \sigma(a), v_{2}^{*}=200, z_{2}^{*}=65.4878$. (See Figure 3.) 

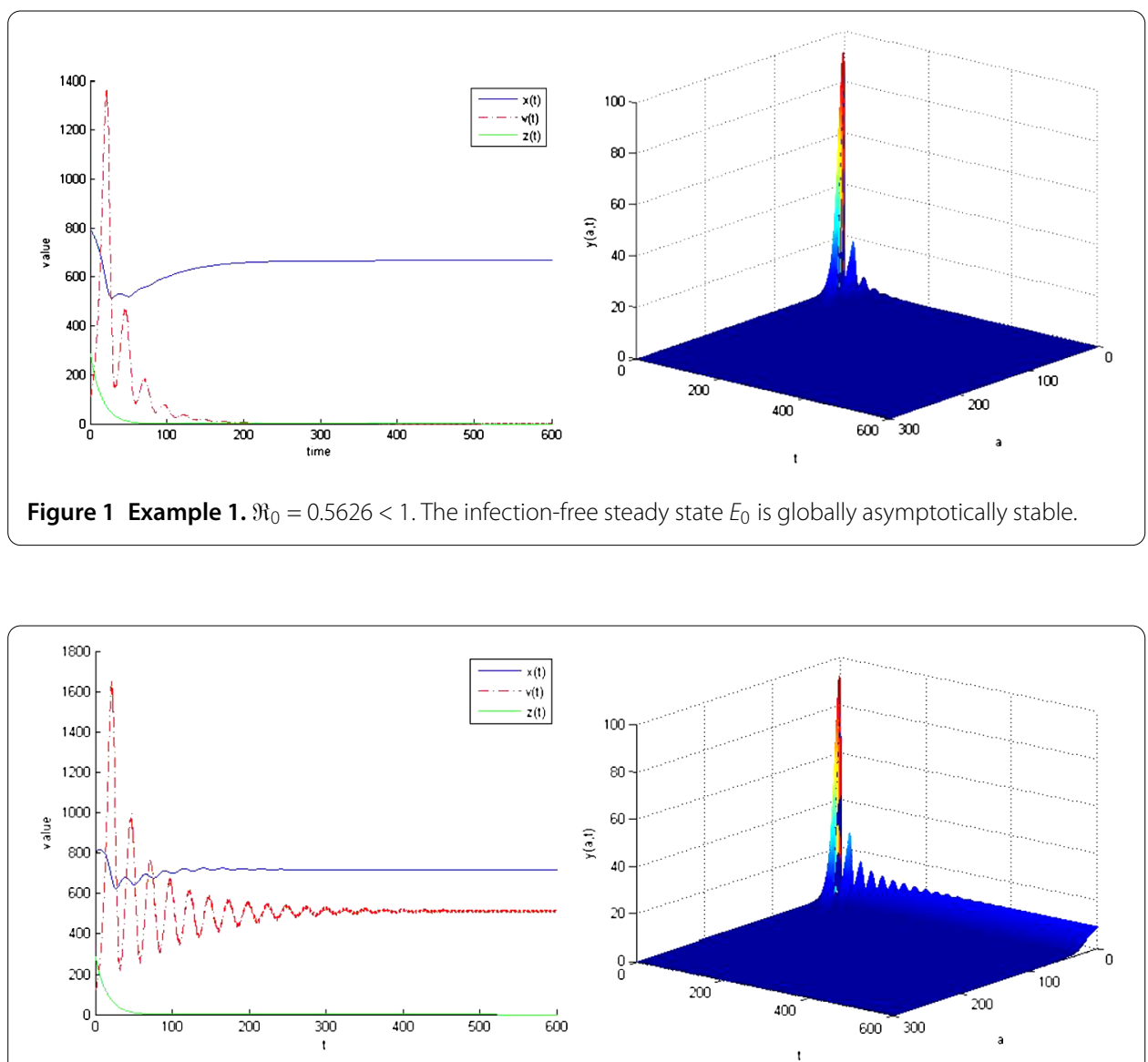

Figure 2 Example $2 . \Re_{0}=1.4064>1, \Re_{1}=0.1153<1$. The immune-inactivated infected steady state $E_{1}^{*}$ is globally asymptotically stable.

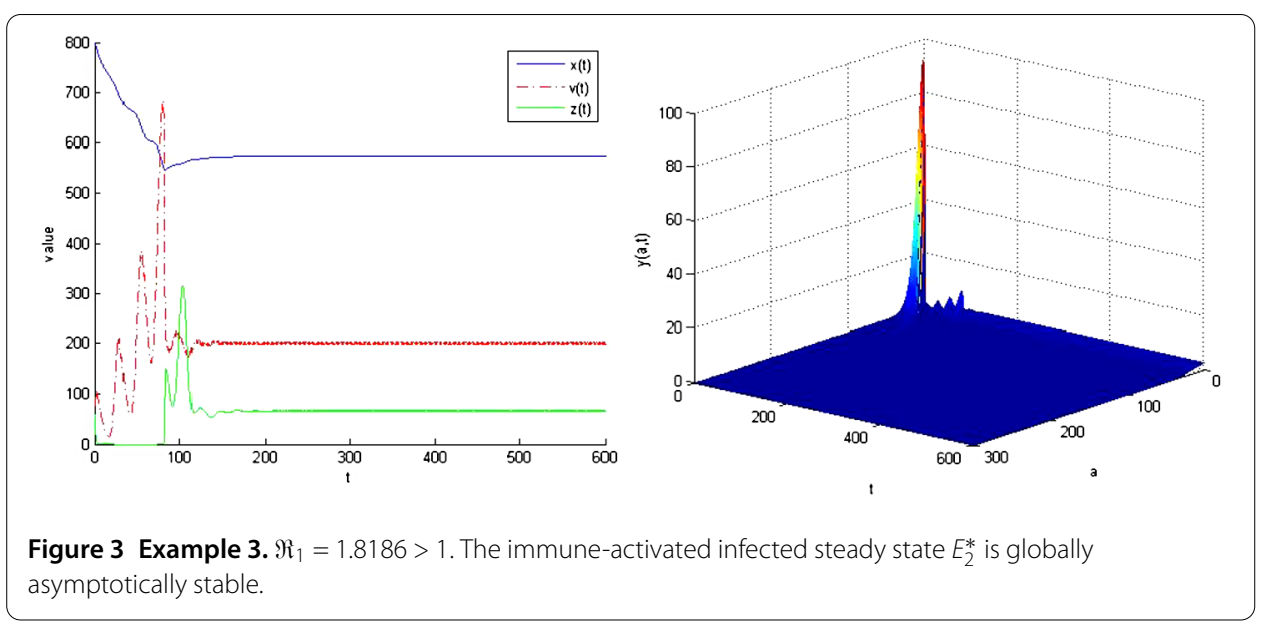

The results of numerical simulation are accord with theorems and the eventual number of each variable in simulation is approximate to the theoretical value. Comparing the results of Example 2 and Example 3 we can find that the densities of infectious free virion and infected $\mathrm{T}$ cells are lower when $\Re_{1}>1$, the humoral immunity is activated. Comparing 
the results of Example 3 and Example 1 we can see that although the humoral immunity is activated, the density of uninfected target T cells of $E_{2}^{*}$ is lower than that of $E_{0}$.

\section{Conclusions}

In this paper, we formulated an in-host viral infection model, in which the influence of humoral immunity and the infection age of the infected cells are considered. Using the method of Lyapunov functionals and LaSalle's invariance principle, we got the conclusions that the global dynamics of the model is determined by the basic reproduction number and the immune-activated reproduction number; if $\Re_{0}<1$, the infection-free steady state is globally asymptotically stable; if $\Re_{1}<1<\Re_{0}$, the immune-inactivated infected steady state is globally asymptotically stable; and if $\Re_{1}>1$, the immune-activated infected steady state is globally asymptotically stable. Comparing the expressions of immuneinactivated infected steady state with immune-activated infected steady state, we saw that $v_{1}^{*}-v_{2}^{*}=\left(\Re_{1}-1\right) \frac{c d u+\beta b u}{\beta c u}$, so humoral immunity has a positive role in the reduction of the virus. Moreover, analyzing the immune-activated reproduction number $\Re_{1}$, we found the parameter $c$ (the birth rate of $B$ cells) effects the value of $\Re_{1}$ a lot. The birth rate of $B$ cells reflects the sensibility of humoral immunity to the virus; generally speaking it can be greatly improved by vaccination.

To illustrate the theoretical results, we did some numerical simulations. Our simulation results confirmed the analytic results. Figures 1-3 showed the stability of $E_{0}, E_{1}^{*}, E_{2}^{*}$, respectively, and by comparison we saw the effect of humoral immunity in the reduction of virus.

\section{Competing interests}

The authors declare that they have no competing interests.

\section{Authors' contributions}

All authors contributed equally to the writing of this paper. All authors read and approved the final manuscript

\section{Acknowledgements}

This work was supported by the National Natural Science Foundation of China (Nos. 11371368, 11071254) and the Natural Science Foundation of Hebei Province (No. A2014506015).

Received: 8 June 2015 Accepted: 19 December 2015 Published online: 07 January 2016

References

1. Deans, JA, Cohen, S: Immunology of malaria. Annu. Rev. Microbiol. 37, 25-49 (1983)

2. Inoue, T, Kajiawara, T, Sasaki, T: Global stability of models of humoral immunity against multiple viral strains. Journal of Biological Dynamics 4, 282-295 (2010)

3. Kajiwara, T, Sasaki, T: A note on the stability analysis of pathogen-immune interaction dynamics. Discrete Contin. Dyn. Syst., Ser. B 4, 615-622 (2004)

4. Nowak, MA, Bangham, CRM: Population dynamics of immune responses to persistent viruses. Science $272,74-79$ (1996)

5. Murase, A, Sasaki, T, Kajiwara, T: Stability analysis of pathogen-immune interaction dynamics. J. Math. Biol. 51, 247-267 (2005)

6. Perelson, AS: Modelling viral and immune system dynamics. Nat. Rev. Immunol. 2, 28-36 (2002)

7. Wang, S, Zou, D: Global stability of in-host viral models with humoral immunity and intracellular delays. Appl. Math. Model. 36, 1313-1322 (2012)

8. Nelson, PW, Gilchrist, MA, Coombs, D, Hyman, JM, Perelson, AS: An age-structured model of HIV infection that allow for variations in the production rate of viral particles and the death rate of productively infected cells. Math. Biosci. Eng. 1, 267-288 (2004)

9. Reilly, C, Wietgrefe, S, Sedgewick, G, Haase, A: Determination of simmian immunodeficiency virus production by infected activated and resting cells. AIDS 21, 163-168 (2007)

10. Gilchrist, MA, Coombs, D, Perelson, AS: Optimizing within-host viral fitness: infected cell lifespan and virion production rate. J. Theor. Biol. 229, 281-288 (2004)

11. Huang, G, Liu, X, Takeuchi, Y: Lyapunov functions and global stability for age-structured HIV infection model. SIAM J. Appl. Math. 72, 25-38 (2004)

12. Wang, J, Zhang, R, Kuniya, T: Mathematical analysis for an age-structured HIV infection model with saturation infection rate. Electron. J. Differ. Equ. 2015, 33 (2015)

13. Hale, JK, Lunel, SV: Introduction to Functional Differential Equations. Springer, New York (1993) 